\title{
CORRESPONDENCE
}

\author{
Southampton's 10th anniversary

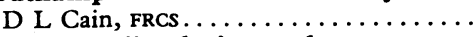 \\ Gall-stone dissolution and recurrence: \\ are we being misled? \\ G J De Lacey, FRCR, and H B Meire, FRCR; \\ $F$ W Wright, FRCR; R H Dowling, FRCP, and \\ D C Ruppin, мB................ 131 \\ Nine months' chemotherapy for \\ pulmonary tuberculosis \\ K M Citron, FRCP. \\ Increase in antibiotic resistance in \\ Haemophilus influenzae in the \\ United Kingdom since 1977 \\ R J Fallon, FRCPATH. . \\ Severe bleeding disorders in children \\ with normal coagulation screening \\ tests \\ R M Hardisty, FRCPATH; P J Green \\ MRCPATH ; P J Darbyshire, MRCP, and O B \\ Eden, MRCP; S T S Durrant, MB .......... \\ Abolish course organisers in general \\ practice \\ D M Malins, MRCP
}

Tetanus surveillance and prophylaxis

D L Hadley, BM................ 134

Side effects of benoxaprofen

J A Hicklin, FRCPED; W Fine, FRCP; J D

Etherington, MRCGP; E Caroline Dunn,

MRCP; L Wibell, MD, and L Benson, MD;

M F Shadforth, MRCP; P Emery, MRCP, and

R Grahame, FRCP.............. 135

Specificity of the immunosuppressive action of carbimazole in Graves's disease

J How, MRCP, and others. .

Xylene-induced epilepsy following

innocent glue sniffing

T Coates, MFoм............... 137

Auditing surgical mortality in elderly patients

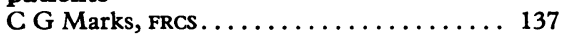

Laboratory accuracy

Janice Went, MRCPATH............ 138
Career structure

Barbara A Thornley, frarcs......... 138

Work load of consultants

K R Llewellin, FRCP............ 138

Pathology and university cuts

R C Curran, PRCPATH............. 138

Points Side effects of benoxaprofen (D A Fenton and J S English); Unusual complication of perforated appendix (K Cronin); Cardiac catheterisation: here today, where tomorrow? (D M Krikler); A plain man's guide to the management of migraine (M G Zambellas); Elective surgery after myocardial infarction (F J M Walters); Regular prescribing in a residential home for elderly women (Jeannie E Stirrat); A happier old age in Denmark? (D N Slater); Payment for notification of infectious diseases $(\mathrm{J} H$ Hardiman); Materia Medica Ecclesiastica (R Greene); Big versus small maternity units (J E U Moxon) ................ 139

\begin{abstract}
We may return unduly long letters to the author for shortening so that we can offer readers as wide a selection as possible. We receive so many letters each week that we have to omit some of them. Letters should be typed with double spacing between lines and must be signed personally by all their authors. We cannot acknowledge their receipt unless a stamped addressed envelope or an international reply coupon is enclosed.

Correspondents should present their references in the Vancouver style (see examples in these columns). In particular, the names and initials of all authors must be given unless there are more than six, when only the first three should be given, followed by et al; and the first and last page numbers of articles and chapters should be included. Titles of papers are not, however, included in the correspondence section.
\end{abstract}

\section{Southampton's 10th anniversary}

SIR,-As a graduate of Southampton University Medical School, I found your leading article on Southampton's 10th anniversary (12 June, p 1730) of great interest. I would like to raise some points.

It is difficult to be objective in assessing the success of the new medical school in its output of doctors. What criteria can be used to assess the ability of any group of doctors? Should one take into account the number of graduates who have gained a higher qualification, or, alternatively, the numbers that have been sued for malpractice by their patients. Clearly neither of these are good indicators on how good or bad a doctor is. Probably the best method of assessment is the impression created on other doctors, and in this respect it is reassuring that most clinical teachers in the district general hospitals in the Wessex region consider Southampton graduates to be at least as good as those from other medical schools, although these teachers are likely to be biased in favour of Southampton graduates.

My own impression of Southampton graduates is that the best graduates are as good as those found anywhere and equally the worst graduates are as bad as those found anywhere. I think it is impossible to say at this early stage what the general ability of those qualifying from Southampton is compared with other graduates.

My own impression of the course was that it was certainly enjoyable, and I think much may be learned by "conventional" medical schools from the Southampton course. In particular, one of the best features I think is the "farming out" of medical students in their final year to district general hospitals in the region, spending only a small proportion of their time in the teaching hospital itself.

The main advantages of this are that common conditions are seen by the medical student rather than the rare conditions that tend to congregate in academic units within teaching hospitals, and there is a good teacherto-student ratio. Often there is only one medical student on a firm of three to four medical staff and this means that he has more attention paid to him for teaching, and he is frequently allowed to act as a "mini-houseman" but under close supervision.

The fourth-year project, which takes up most of the student's time in the fourth year, is certainly controversial, and in my opinion it is not necessary to spend such a large proportion of undergraduate time doing research in depth within a very narrow field. There is a wide diversity of opinion, however, among Southampton's graduates on the value of this study in depth.

The thing one remembers most from the teaching at Southampton was the enthusiasm and real interest that the teachers showed from preclinical through to clinical work.

Your leading article suggests that there was an unexplained reluctance to enter surgery; I would disagree with this. Of the hundred or so graduates who have been qualified long enough to be eligible for the fellowship regulations, there are five who are fellows of the Royal College of Surgeons, and I know of several more graduates who are following a career in surgery but are not yet eligible to take the examinations.

Your article also mentioned that there was reluctance to enter obstetrics and gynaecology, and as far as I am aware there are no graduates from my own cohort of students who have entered this field, but this is by no means unique among medical schools, as Parkhouse's studies on career preferences of graduates qualifying in Britain have shown.

At present the difficult problem of defining criteria by which graduates may be assessed is yet to be overcome, but if it is, and Southampton graduates are shown to be as good as-or better than-graduates from other medical schools, then the curriculum of every medical school in the country must be reviewed.

David CaIN

St Richard's Hospital,

West Sussex PO19 4SE

Gall-stone dissolution and recurrence: are we being misled?

SIR,-Dr G M Fraser and others (19 June, p 1873) raise an important point in relation to the relative accuracy of oral cholecystography and ultrasound. There are indeed important 
clinical and staffing implications if the findings of Dr K W Somerville and colleagues (1 May, p 1295) are readily reproducible in patients suspected of gall-bladder disease.

In this regard it is disturbing that scrutiny of 15 comparative studies between 1976 and 1980 showed that 12 out of 15 included ultrasound technical details, but only one included important details regarding the oral cholecystogram. Most studies provided no information whatsoever, not even as to the contrast medium. This includes five which recommend or suggest that ultrasound should be the investigation of choice in detecting gall stones. ${ }^{1-5}$

In their important paper Dr Somerville and colleagues did not describe the cholecystographic technique but subsequently (19 June, p 1873) stated that erect compression films were not routinely included. It is possible that good ultrasound is more accurate than a good oral cholecystogram, but it is arguable that the evidence in support of this has overwhelmingly been a comparison between enthusiastic state-of-the-art ultrasound and out-of-date oral cholecystographic technique. It is worth remembering that the oral cholecystogram, properly carried out, is a simple, inexpensive, and highly accurate procedure ${ }^{6}$ and it is essential that if it is to be abandoned this important change in diagnostic practice be based on good data.

The prospective study that $\mathrm{Dr}$ Somerville and colleagues are seeking is presently being carried out at this institution, and we will be reporting our findings later this year. A preliminary analysis of our first 200 patients, however, indicates an oral cholecystography false-negative rate of $1.0 \%$ for stones but an ultrasound false-negative rate which might be as high as $4.0 \%$, being made up of stones $(1.0 \%)$ and cholesterolosis/adenomyomatosis (3\%).

Perhaps other comparative surveys, which are obviously necessary, will look critically at, and provide details of, the technique of oral cholecystography.

GeRALD DE LACEY HyLTON MEIRE

Department of Radiology and Ultrasound,

Northwick Park Hospital,
Harrow, Middx HA1 3UJ

'Woolson AH, Goldberg BB, fAMA 1978;240: 2 Cooperberg PL, Burhenne HJ. N Engl f Med 1980; 302:1277-9.9. Penney HF. Radiology 1980;136:
725tosh DM,
725-7.

Stone KS, Scholte

Y Yas W, Salem S. I Can Assoc Rad 1980;31:116.

Krook PM, Allen FH, Bush WH, Malmer G, Macleane MD. Radiology 1980;135:145-8.

SIR, - I was interested to read the letter of Dr G M Fraser and others and the reply by Dr K W Somerville and others (19 June, p 1873). The former were questioning the findings of the latter, particularly with regard to their technique of cholecystography.

Reading the reply, I think that their criticisms were indeed valid. The gall bladder, which concentrates excreted contrast medium in the bile by removal of water, acts as a density column, which is why erect views should be taken first before the density column is disturbed by the patient lying down and particularly being turned into the prone position. I would consider that routine fluoroscopy, with compression (and elevation of the right breast, if large, in women), is essential to see a thin horizontal line of "floating" gall stones. I agree that this cannot be left to over-couch views by a radiographer. Straying into the diagnosis of gall stones de novo it often appears now to be the view that ultrasound is infallible and cholecystography fallible, but what about the spurious bowel shadows seen with ultrasound that mimic gall stones? Furthermore, there is a great danger that if a barium meal is not combined with a cholecystogram, as happens in many centres (the cholecystogram spot films taking only a few moments of the radiologist's time), then many gastric or duodenal lesions will be missed. None of us are perfect on really determining by history whether the gall bladder or the upper gastrointestinal tract - that is, stomach or duodenum -is at fault, and with "open access" the combined approach is even more important.

Please do not get me wrong, I am not against ultrasound. Indeed, I practice it, but let us use it intelligently for the gall bladder, in combination with cholecystography and the barium meal.

Churchill Hospital,

F W WRIGHT Headington,
Oxford

SIR,-In their recent paper (1 May, p 1295) entitled "Gall-stone dissolution and recurrence: are we being misled ?" $\mathrm{Dr} \mathrm{K} W$ Somerville and colleagues ask an important question. In attempting to answer it, they compare cholecystographic and real-time ultrasonographic findings in 14 patients who had apparently shown complete gall-stone dissolution, as judged by two "adequate" cholecystograms performed three months apart, during continued medical treatment. They found that gall-bladder ultrasound showed "definite evidence," by which they mean "an echogenic focus within the gall bladder, posterior acoustic shadowing and dependent movement of the echogenic focus with gravity," of at least one small stone in the gall bladder in six of the 14 patients who had had "negative" oral cholecystograms. They also studied 10 further patients who had previously been shown, again by cholecystography, to have confirmed complete gall-stone dissolution $^{1}$ and who were receiving postdissolution maintenance treatment. Only one of these 10 showed stones on repeat oral cholecystography but cholecystosonography detected gall stones in seven of the 10 patients. At first sight, therefore, it might seem that ultrasound of the gall bladder is more reliable than oral cholecystography in assessing the response to medical treatment and in detecting possible gall-stone recurrence. But before we too readily accept Somerville's suggestion that "complete dissolution of gall stones on cholecystography may thus be apparent rather than real," some comments seem indicated.

Firstly, when attempting to detect tiny stones with either technique, one is working at, or beyond, the limits of resolution. Both methods have a certain number of false-positive and false-negative results. In the context of gall-stone dissolution, therefore, the critical question relates to whether or not cholecystography has more so-called false-negative results than ultrasonography and, in the context of detecting possible gall-stone recurrence, whether or not ultrasound examinations yield more false-positive results than radiography. Unfortunately, there is no absolute reference standard and in the absence of definitive proof (by examining gall-bladder contents removed at surgery or necropsy) comparison of results obtained using the two indirect methods can neither validate nor invalidate the findings of either technique. Unfortunately, it is difficult to imagine a clinical situation where such proof might be forthcoming. Having embarked on medical treatment as an alternative to surgery and having apparently dissolved the patients' gall stones, it is hardly ethical then to propose elective cholecystectomy simply to verify, or otherwise, the cholecystographic or ultrasonographic findings. The only conceivable situation where such proof might be forthcoming is in patients who, after gall-stone dissolution therapy, are left with non-dissolvable gravel, sand, or sludge in the gall bladder which is detected by ultrasound but not by cholecystography. In that situation, cholecystectomy could be recommended but as yet no such studies have been carried out.

Secondly, given the fact that ultrasonography is both operator- and machine-dependent, it is difficult if not impossible to compare the results obtained by cholecystosonography with those obtained by oral cholecystography. There have, of course, been many previous attempts to do so. ${ }^{2-4}$ But in detecting the presence of gall stones, can one reasonably compare the accuracy of routine cholecystographic films (usually taken by a radiographer and seldom requiring more than a few minutes of a radiologist's time for their interpretation) with ultrasonographic results obtained by individuals who have varying degrees of enthusiasm and experience, who spend varying amounts of time over their examination, and who use equipment with varying degrees of sophistication? One is sometimes concerned that claims in favour of the diagnostic efficacy of ultrasound may have been biased by the effort and time put into the examination by skilled observers. In theory, such bias might be partially corrected if both radiographs and ultrasonographic films were analysed retrospectively for intraobserver and interobserver error. Unfortunately, Dr Somerville and colleagues do not say whether their cholecystogram and ultrasound films were assessed blindly, on more than one occasion, and by independent observers.

We should emphasise that we do not wish to detract from ultrasonography in the diagnosis of gall stones. We too think that it is a safe, sensitive, and acceptable technique which provides a valuable addition to oral cholecytography. But in the specific area of gall-stone dissolution we believe that at present ultrasonography cannot replace cholecystography when patients are being selected for medical treatment and in monitoring their response to therapy.

It is universally agreed that there are two essential prerequisites to selecting patients for gall-stone dissolution therapy-the cystic duct should be patent (as judged by opacification of the gall bladder during oral cholecystography) and the gall stones radiolucent. Since ultrasound cannot distinguish between radio-opaque and radiolucent stones and cannot show cystic duct obstruction, cholecystography is still necessary to establish that these two requirements have been met before starting gall-stone patients on medical treatment.

Ultrasound may have a place in monitoring the response to dissolution therapy but again it cannot detect calcification acquired during bile acid therapy-which has been seen during treatment with chenodeoxycholic acid ${ }^{5-6}$ and particularly with ursodeoxycholic acid. ${ }^{7-9}$ In theory, acquired calcification could be detected if plain radiographs of the gall-bladder area were taken routinely to supplement the ultrasonographic examinations. In practice, however, the presence of overlying bowel gas, of calcified costal carilages, or of miscellaneous, non-gall-stone calcification means that it is often difficult to diagnose acquired gall-stone calcification on plain $x$-ray film. Furthermore, in our experience 710 up to $10 \%$ of patients may develop a "non-functioning" (non-opacifying) gall bladder during dissolution therapy - often silently in the absence of overt clinical symptoms. Ultrasound cannot detect this complication, and since cystic duct obstruction precludes further gall-stone dissolution if one relied on ultrasonography alone to monitor the response to 